\title{
Pulmonary Tuberculosis in Severely-malnourished or HIV-infected Children with Pneumonia: A Review
}

\author{
Mohammod Jobayer Chisti',2, Tahmeed Ahmed², Mark A.C. Pietroni', \\ Abu S.G. Faruque ${ }^{2}$, Hasan Ashraf ${ }^{1,2}$, Pradip K. Bardhan ${ }^{1,2}$, Md. Iqbal Hossain ${ }^{1,2}$, \\ Sumon Kumar Das', Mohammed Abdus Salam ${ }^{3}$ \\ 'Clinical Services, ${ }^{2}$ Centre for Nutrition \& Food Security, ${ }^{3}$ Research Administration, icddr,b, \\ GPO Box 128, Dhaka 1000, Bangladesh
}

\begin{abstract}
Presentation of pulmonary tuberculosis (PTB) as acute pneumonia in severely-malnourished and HIVpositive children has received very little attention, although this is very important in the management of pneumonia in children living in communities where TB is highly endemic. Our aim was to identify confirmed TB in children with acute pneumonia and HIV infection and/or severe acute malnutrition (SAM) (weight-for-length/height or weight-for-age $\mathrm{z}$ score $<-3$ of the WHO median, or presence of nutritional oedema). We conducted a literature search, using PubMed and Web of Science in April 2013 for the period from January 1974 through April 2013. We included only those studies that reported confirmed TB identified by acid fast bacilli (AFB) through smear microscopy, or by culture-positive specimens from children with acute pneumonia and SAM and/or HIV infection. The specimens were collected either from induced sputum (IS), or gastric lavage (GL), or broncho-alveolar lavage (BAL), or percutaneous lung aspirates (LA). Pneumonia was defined as the radiological evidence of lobar or patchy consolidation and/or clinical evidence of severe/ very severe pneumonia according to the WHO criteria of acute respiratory infection. A total of 17 studies met our search criteria but 6 were relevant for our review. Eleven studies were excluded as those did not assess the HIV status of the children or specify the nutritional status of the children with acute pneumonia and TB. We identified only 747 under-five children from the six relevant studies that determined a tubercular aetiology of acute pneumonia in children with SAM and/or positive HIV status. Three studies were reported from South Africa and one each from the Gambia, Ethiopia, and Thailand where 610, 90, 35, and 12 children were enrolled and $64(10 \%), 23(26 \%), 5(14 \%)$, and $1(8 \%)$ children were identified with active TB respectively, with a total of 93 (12\%) children with active TB. Among 610 HIV-infected children in three studies from South Africa and 137 SAM children from other studies, 64 (10\%) and 29 (21\%) isolates of M. tuberculosis were identified respectively. Children from South Africa were infected with HIV without specification of their nutritional status whereas children from other countries had SAM but without indication of their HIV status. Our review of the existing data suggests that pulmonary tuberculosis may be more common than it is generally suspected in children with acute pneumonia and SAM, or HIV infection. Because of the scarcity of data, there is an urgent need to investigate PTB as one of the potential aetiologies of acute pneumonia in these children in a carefully-conducted larger study, especially outside Africa.
\end{abstract}

Key words: Acute pneumonia; Children; HIV; Severe malnutrition; Tuberculosis

Correspondence and reprint requests:

Dr. Mohammod Jobayer Chisti

Scientist (CNFS) and Clinical Lead, ICU

and Consultant Physician, ARI Ward

Dhaka Hospital, icddr,b

68 Shaheed Tajuddin Ahmed Sarani

Mohakhali, Dhaka 1212

Bangladesh

Email: chisti@icddrb.org

Fax: + (880-2) 8823116 and 9885657

\section{INTRODUCTION}

According to the WHO global tuberculosis report 2012, there were an estimated 8.7 million new cases of TB (13\% co-infected with HIV) and 1.4 million people died of $\mathrm{TB}$, including 9,90,000 deaths among HIV-negative individuals and 430,000 among people who were HIV-positive in 2011 (1). However, among the overall global estimates, the 
incidence of active TB in children (defined as those aged $<15$ years) was estimated at 490,000 cases, and the total number of deaths from TB among HIVnegative cases was estimated at 64,000 in 2011 (1). Data from recently-published articles $(2,3)$ suggest that, with accurate diagnosis and good reporting system, children younger than 15 years are likely to contribute $10-20 \%$ of the total disease burden due to $\mathrm{TB}$ in endemic areas, with a TB incidence estimated at around $50 \%$ of that recorded in adults (2). Mortality from pulmonary TB (PTB) is high among children who present with acute pneumonia and severe acute malnutrition (SAM) (4) with or without HIV infection (5). The co-morbidities from acute pneumonia with SAM or acute pneumonia with HIV are a serious problem among under-five children in developing countries (5-7). The duration of symptoms in PTB presenting as acute pneumonia, with cough, fever, anorexia, and failure to thrive, are often less than two weeks. A recentlypublished systematic review reported that, in addition to the usual respiratory bacterial aetiology in community-acquired acute pneumonia in severelymalnourished children, there are other important causes that remain mostly unexplored, and one of these causes is TB (8). Yet, presentation of PTB as acute pneumonia in severely-malnourished and HIV-infected children has received very little attention, although this is important in the management of pneumonia in children living in communities where $\mathrm{TB}$ is highly endemic (8).

\section{Epidemiology and stratification of risks}

The epidemiology of childhood TB with acute pneumonia, irrespective of nutritional or HIV status, has not been well-addressed in medical literature $(8,9)$. In general, children have paucibacillary disease and reduced strength of coughing and rarely contribute to disease transmission compared to adults $(10,11)$. Children with acute pneumonia and severe malnutrition and/or HIV infection may not be different in these characteristics. The natural history of the disease suggests age as the most important risk factor (12). The risk of disease after primary infection with TB is as high as $50 \%$ in infants below the age of one year, $10-20 \%$ in children aged $1-2$ year(s), $5 \%$ in children aged $2-5$ years, and only $2 \%$ in children aged 5-10 years. The risk increases to $10-20 \%$ for children older than 10 years $(11,13)$. As in infants, severe malnutrition and HIV infection are also serious risk factors in children $(5,14)$. All of these risk factors are associated with poor cell-mediated immune responses resulting in severe forms of disease after infection with TB (15-17). Of the infected children who progress to disease, approximately $95 \%$ will develop disease within 12 months of infection (18). Thus, on the basis of the high exposure rates of TB in endemic countries, all under-five children with SAM and/or HIV infection with acute pneumonia should be categorized as a high-risk group in terms of developing PTB.

In this review, we aimed to examine the aetiologic role of PTB in children with SAM and/or HIV infection presenting with features of acute pneumonia.

\section{MATERIALS AND METHODS}

We conducted a literature search, limited to the English language, to identify reports focusing on tuberculosis in children with SAM and/or HIV infection with acute pneumonia. Severe malnutrition was defined as weight-for-age or weight-for-height $z$ score $<-3$ of the median of the National Center for Health Statistics (NCHS) (19). We have also included the children with marasmus, or kwashiorkor, or marasmic-kwashiorkor defined according to the Wellcome classification (20). Acute pneumonia was defined as the radiological evidence of lobar or patchy consolidation (6) and/or clinical evidence of severe/very severe pneumonia according to the WHO criteria of acute respiratory infection (21). The databases searched in April 2013 included: PubMed and Web of Science (January 1974 through April 2013). Table 1 summarizes the search strategies and outcomes. All identified abstracts were reviewed, full articles were retrieved and evaluated for those abstracts that suggested potential relevance to our purpose. We included studies reporting culture-positive TB or acid fast bacilli (AFB) by smear microscopy from samples obtained from spontaneous/induced sputum (IS) or gastric lavage (GL), or broncho-alveolar lavage (BAL), or percutaneous lung aspirates (LA) in under-five children with acute pneumonia and SAM and/or positive HIV status.

\section{RESULTS}

A total of 17 studies met our search criteria. Of these, only 6 published studies $(4,22-26)$ relevant to our review provided a total of 747 children (Table 1). The remaining 11 publications (27-37) were excluded as those evaluated the tubercular aetiology of acute pneumonia either without reporting their HIV status or without specifying their nutritional status. One study (38) was excluded from the review because the children were not enrolled in the study due to acute pneumonia, rather acute pneumonia was reported as a finding among a cohort of culture-confirmed TB. 
Table 2 summarizes the review results showing six reports, including three from South Africa, one each from the Gambia, Ethiopia, and Thailand that focused on children younger than 5 years. Studies other than those from South Africa $(4,25,26)$ used mainly LA for the isolation of M. tuberculosis. Three studies (22-24) from South Africa used mainly BAL or GL, one of these (23) also used IS for the isolation. The diagnosis of PTB was based on identification of M. tuberculosis by culture in all studies, except the study from the Gambia where three of their 5 cases of PTB were diagnosed by the identification of AFB only, one by AFB and culture, and the remaining one by culture of $M$. tuberculosis only. The total number of $M$. tuberculosis isolates in all reports combined was 93 out of 747 (12\%). There were 610 HIV-infected children in studies from South Africa and 137 SAM children from other studies; 64 out of $610(10 \%)$ HIV-infected children and 29 out of 137 (21\%) SAM children were found to have M. tuberculosis. Data on CD4 count of HIV-infected children were not available. Nutritional status of children with $M$. tuberculosis was not specified in
South African studies, and HIV status of children in other studies was not mentioned.

\section{DISCUSSION}

An observation of overall 12\% cases of TB in 747 children with acute pneumonia with SAM or positive HIV status is a very important finding for clinicians in developing countries where the co-morbidity of acute pneumonia and SAM, or positive HIV status is common. The possibility of TB is not often considered in the differential diagnosis of acute pneumonia and, therefore, work-up for TB is not considered in such cases. Pulmonary TB is common in HIV-infected children, potentially due to their reduction in immunity $(5,38)$, which might help the progression of latent TB infection to active TB (5). With the increased uptake of pneumococ$\mathrm{cal}$ and $H$. influenzae type $\mathrm{b}$ vaccine in developing countries, these pathogens will become relatively less important as causative agents of acute pneumonia (39-41) while M. tuberculosis might emerge more frequently as an isolated pathogen in children

\begin{tabular}{|c|c|c|c|c|}
\hline Database & Strategy and key words used & $\begin{array}{l}\text { Total number } \\
\text { of matches }\end{array}$ & $\begin{array}{l}\text { Publications } \\
\text { relevant for } \\
\text { review }\end{array}$ & Year of publications \\
\hline PubMed & $\begin{array}{l}\text { (pulmonary OR lung) AND (tu- } \\
\text { berculosis OR TB) AND (infant } \\
\text { OR child }{ }^{\star} \text { OR (paediatric OR } \\
\text { pediatric) AND (pneumonia OR } \\
\text { (acute lower respiratory infec- } \\
\text { tion) OR ALRI) }\end{array}$ & 455 & 6 & $\begin{array}{c}\text { January1974 to } \\
\text { April } 2013\end{array}$ \\
\hline $\begin{array}{l}\text { Web of } \\
\text { Science }\end{array}$ & $\begin{array}{l}\text { Tuberculosis AND Pneumonia } \\
\text { AND (infant OR child OR pedi- } \\
\text { atric OR paediatric) }\end{array}$ & 323 & 6 & $\begin{array}{c}\text { January1974 to } \\
\text { April } 2013\end{array}$ \\
\hline
\end{tabular}

\begin{tabular}{|c|c|c|c|c|c|}
\hline Author and year of publication & Country & $\begin{array}{c}\text { Age } \\
\text { (months) }\end{array}$ & $\begin{array}{c}\text { No. of } \\
\text { patients }\end{array}$ & $\begin{array}{l}\text { No. } \\
\text { (\%) of } \\
\text { isolates }\end{array}$ & $\begin{array}{l}\text { Main source } \\
\text { of sample }\end{array}$ \\
\hline \multicolumn{6}{|l|}{ With HIV } \\
\hline McNally LM et al., 2007 & South Africa & $1-59$ & 87 & $18(21)$ & BAL \\
\hline Zar HJ et al., 2000 & South Africa & $<60$ & 100 & $10(10)$ & IS and GL \\
\hline Madhi SA et al., 2000 & South Africa & $2-60$ & 423 & $36(9)$ & GL \\
\hline \multicolumn{6}{|l|}{ Without HIV } \\
\hline Adegbola et al., 1994 & Gambia & $3-58$ & 35 & $5(14)$ & LA and IS \\
\hline Shimeles D and Lulseged S, 1994 & Ethiopia & $4-60$ & 90 & $23(26)$ & LA \\
\hline Morehead et al., 1974 & Thailand & $10-50$ & 12 & $1(8)$ & LA \\
\hline
\end{tabular}


with acute pneumonia in TB-endemic countries, especially those having SAM and/or positive HIV status. On the other hand, once children become infected with $\mathrm{TB}$, this may progress more rapidly into PTB in them compared to other forms of TB $(5,38)$. However, given the natural history of childhood TB, it is very unlikely that TB will result in severe progressive pathology of lungs with features of acute pneumonia. Moreover, children with TB often fail to gain weight and become symptomatic with features of malnutrition. In our review, children with SAM without the mention of HIV status might also have reduced immune status almost similar to that in HIV, and they have a reduced ability to clear infecting organisms, have reduced mucosal immunity and a favourable microenvironment at the point of organism deposition (42), leading to a higher risk of pulmonary co-infection (43). At this point, due to co-infections in the respiratory system, disease progression might become more severe, and children potentially might present with features of acute pneumonia (5). Although the effects of cell-mediated immunity are different in HIV-infected children compared to SAM children, these two conditions are still the risk factors in developing active $\mathrm{TB}$ with potential severe ramification in under-five children, especially in TB-endemic settings $(4,36)$. The role of PTB in children with acute pneumonia having any of the above conditions is still unclear.

The higher (21\%) identification of PTB in three of the publications included in this review based on percutaneous lung aspirates done in children with SAM without any adverse events is another important finding in this review $(4,25,26)$. It is not common in countries outside Africa, with higher likelihood of missing TB at the time of diagnosis $(29,44)$. Bacteriological diagnosis of pulmonary $\mathrm{TB}$ is very difficult in a resource-poor setting where acute pneumonia and SAM are very common and where there is lack of skilled personnel and adequate facilities to perform lung puncture, although the complication from this procedure is very rare $(29,44)$. Moreover, the gold standard of diagnosis—culture confirmed TB-is of limited use in severely-malnourished children due to the paucibacillary nature of the disease and poor bacteriologic yields as cavitation happens rarely in the expansion of the primary focus in malnourished children (45). However, the diagnosis of childhood TB in children in developing countries often employs widely-used scoring systems, such as Modified Kenneth Jones criteria (45) and/or WHO criteria published in 2006 (46), which are based on clinical and radiological findings and TST. They often produce inconsistent diagnosis, especially in children in HIV-endemic areas (47). However, the rate of HIV infection in patients with TB has so far remained below 1\% in Bangladesh, China, Indonesia, and Pakistan, although it is more common in severely-malnourished children with TB in sub-Sahara and Africa (48). This indicates the potential value and the need for sensitive bacteriology-based diagnostic approaches, particularly in endemic areas where children frequently present with advanced disease (11). The laboratory in the Medical Research Council (MRC) in the Gambia performs lung puncture with a relatively high yield of organisms (4), and it is presently used in a global multicentre pneumonia aetiology research in child health (PERCH) study (49). In this context, with available facilities for careful monitoring and managing complications effectively by trained personnel in children with acute pneumonia and SAM and/or HIV infection, who do not produce sputum, percutaneous lung aspiration would be the diagnostic test of choice for a better isolation rate of TB. This approach potentially promotes early initiation of antitubercular therapy which ultimately helps reduce morbidity and deaths in such populations.

\section{Limitations}

Our review is limited by the fact that we could identify only six relevant publications covering a relatively-small number of children with a tubercular aetiology of acute pneumonia, along with SAM or HIV infection, which might not be mutually exclusive. We were unable to use other publications on the diagnosis of tuberculosis in children with acute pneumonia because of the lack of information on the specific nutritional status or HIV status of the children. This might have led to an underestimation of the actual frequency of PTB in SAM and/or HIV-infected children with acute pneumonia. Moreover, lack of information on CD4 count of HIV-infected children is another limitation of this review, which makes it difficult to determine the extent of immune deficiency in the HIV-infected children. However, the combination of two groups of children (children with HIV infection or SAM) in evaluating burden of PTB in our review may also be a limitation of this review.

\section{Conclusions}

Our review suggests that PTB may be a common cause of acute pneumonia in severely-malnourished or HIV-infected under-five children. The identification of high frequency of PTB in severelymalnourished or HIV-infected children presenting with acute pneumonia in the reference studies sug- 
gests the need for further investigation of the problem, especially in children outside Africa, to better define the tubercular aetiology of acute pneumonia in such children. The information would certainly play an important role for helping clinicians in making decisions about the diagnosis of PTB in such populations, and policy-makers in amending the management guidelines for acute pneumonia in children with SAM to reduce morbidity and deaths.

\section{REFERENCES}

1. World Health Organization. Global tuberculosis report 2012. Geneva: World Health Organization, 2012. 89 p.

2. Marais BJ, Schaaf HS. Childhood tuberculosis: an emerging and previously neglected problem. Infect Dis Clin North Am 2010;24:727-49.

3. Winston CA, Menzies HJ. Pediatric and adolescent tuberculosis in the United States, 2008-2010. Pediatrics 2012;130:e1425-32.

4. Adegbola RA, Falade AG, Sam BE, Aidoo M, Baldeh I, Hazlett D et al. The etiology of pneumonia in malnourished and well-nourished Gambian children. Pediatr Infect Dis J 1994;13:975-82.

5. Moore DP, Klugman KP, Madhi SA. Role of Streptococcus pneumoniae in hospitalization for acute community-acquired pneumonia associated with cultureconfirmed Mycobacterium tuberculosis in children: a pneumococcal conjugate vaccine probe study. Pediatr Infect Dis J 2010;29:1099-04.

6. Chisti MJ, Ahmed T, Faruque AS, Abdus Salam M. Clinical and laboratory features of radiologic pneumonia in severely malnourished infants attending an urban diarrhea treatment center in Bangladesh. Pediatr Infect Dis J 2010;29:174-7.

7. Chisti MJ, Duke T, Robertson CF, Ahmed T, Faruque AS, Bardhan PK et al. Co-morbidity: exploring the clinical overlap between pneumonia and diarrhoea in a hospital in Dhaka, Bangladesh. Ann Trop Paediatr 2011;31:311-9.

8. Chisti MJ, Tebruegge M, La Vincente S, Graham SM, Duke T. Pneumonia in severely malnourished children in developing countries-mortality risk, aetiology and validity of WHO clinical signs: a systematic review. Trop Med Int Health 2009;14:1173-89.

9. Rudan I, Boschi-Pinto C, Biloglav Z, Mulholland K, Campbell H. Epidemiology and etiology of childhood pneumonia. Bull World Health Organ 2008;86:408-16.

10. Marais BJ, Obihara CC, Warren RM, Schaaf HS, Gie RP, Donald PR. The burden of childhood tuberculosis: a public health perspective. Int J Tuberc Lung Dis 2005;9:1305-13.
11. Marais BJ, Gie RP, Schaaf HS, Beyers N, Donald PR, Starke JR. Childhood pulmonary tuberculosis: old wisdom and new challenges. Am J Respir Crit Care Med 2006;173:1078-90.

12. Chintu C, Mudenda V, Lucas S, Nunn A, Lishimpi $\mathrm{K}$, Maswahu D et al. Lung diseases at necropsy in African children dying from respiratory illnesses: a descriptive necropsy study. Lancet 2002;360:98590.

13. Donald PR, Marais BJ, Barry CE, 3rd. Age and the epidemiology and pathogenesis of tuberculosis. Lancet 2010;375:1852-4.

14. Marais BJ, Gie RP, Schaaf HS, Hesseling AC, Obihara $\mathrm{CC}$, Starke JJ et al. The natural history of childhood intra-thoracic tuberculosis: a critical review of literature from the pre-chemotherapy era. Int $J$ Tuberc Lung Dis 2004;8:392-402.

15. Freiman I, Geefhuysen J, Solomon A. The radiological presentation of pulmonary tuberculosis in children. $S$ Afr Med J 1975;49:1703-6.

16. Madhi SA, Huebner RE, Doedens L, Aduc T, Wesley D, Cooper PA. HIV-1 co-infection in children hospitalised with tuberculosis in South Africa. Int J Tuberc Lung Dis 2000;4:448-54.

17. Hesseling AC, Westra AE, Werschkull H, Donald PR, Beyers N, Hussey GD et al. Outcome of HIV infected children with culture confirmed tuberculosis. Arch Dis Child 2005;90:1171-4.

18. Gedde-Dahl T. Tuberculous infection in the light of tuberculin matriculation. Am J Hyg 1952;56:139214.

19. Jelliffe DB. The assessment of the nutritional status of the community (with special reference to field surveys in developing regions of the world). Monogr Ser World Health Organ 1966;53:3-271.

20. Walker AC. Classification of infantile malnutrition. Lancet 1970;2:1028.

21. World Health Organization. Pocket book of hospital care for children: guidelines for the management of common illnesses with limited resources. Geneva: World Health Organization, 2005. 378 p.

22. Madhi SA, Petersen K, Madhi A, Khoosal M, Klugman $\mathrm{KP}$. Increased disease burden and antibiotic resistance of bacteria causing severe community-acquired lower respiratory tract infections in human immunodeficiency virus type 1-infected children. Clin Infect Dis 2000;31:170-6.

23. Zar HJ, Tannenbaum E, Apolles P, Roux P, Hanslo D, Hussey G. Sputum induction for the diagnosis of pulmonary tuberculosis in infants and young children in an urban setting in South Africa. Arch Dis Child 2000;82:305-8. 
24. McNally LM, Jeena PM, Gajee K, Thula SA, Sturm AW, Cassol S et al. Effect of age, polymicrobial disease, and maternal HIV status on treatment response and cause of severe pneumonia in South African children: a prospective descriptive study. Lancet 2007;369:144051.

25. Morehead CD, Morehead M, Allen DM, Olson RE. Bacterial infections in malnourished children. J Trop Pediatr Environ Child Health 1974;20:141-7.

26. Shimeles D, Lulseged S. Clinical profile and pattern of infection in Ethiopian children with severe proteinenergy malnutrition. East Afr Med J 1994;71:264-7.

27. Kalra SK, Sasidharan T, Vatwani V, Sarkar P. Lung puncture: a diagnostic aid in childhood pneumonia. Indian Pediatr 1981;18:727-30.

28. Escobar JA, Dover AS, Dueñas A, Leal E, Medina P, Arguello A et al. Etiology of respiratory tract infections in children in Cali, Colombia. Pediatrics 1976;57:12330.

29. Falade AG, Mulholland EK, Adegbola RA, Greenwood BM. Bacterial isolates from blood and lung aspirate cultures in Gambian children with lobar pneumonia. Ann Trop Paediatr 1997;17:315-9.

30. Oyedeji GA. The pattern of infections in children with severe protein energy malnutrition. Nigerian J Paediatr 1989;16:55-61.

31. Witte A. [Epidemics return and new ones are added]. Kinderkrankenschwester 1991;10:460-1. [German]

32. Raote GJ, Bhave SY. Clinical profile of measles-a prospective study of 150 hospital based children. Indian Pediatr 1992;29:45-8.

33. Kumar A, Upadhyay S, Kumari G. Clinical presentation, treatment outcome and survival among the HIV infected children with culture confirmed tuberculosis. Curr HIV Res 2007;5:499-504.

34. Peltier CA, Omes C, Ndimubanzi PC, Ndayisaba GF, Stulac S, Arendt V et al. Validation of 2006 WHO prediction scores for true HIV infection in children less than 18 months with a positive serological HIV test. PLoS One 2009;4:e5312.

35. Carrol ED, Mankhambo LA, Guiver M, Banda DL; IPD Study Group, Denis B, Dove W et al. PCR improves diagnostic yield from lung aspiration in Malawian children with radiologically confirmed pneumonia. PLoS One 2011;6:e21042.

36. De Maayer T, Saloojee H. Clinical outcomes of severe malnutrition in a high tuberculosis and HIV setting. Arch Dis Child 2011;96:560-4.
37. Nantongo JM, Wobudeya E, Mupere E, Joloba M, Ssengooba W, Kisembo HN et al. High incidence of pulmonary tuberculosis in children admitted with severe pneumonia in Uganda. BMC Pediatr 2013;13:16.

38. Jeena PM, Pillay P, Pillay T, Coovadia HM. Impact of HIV-1 co-infection on presentation and hospitalrelated mortality in children with culture proven pulmonary tuberculosis in Durban, South Africa. Int J Tuberc Lung Dis 2002;6:672-8.

39. Martin M, Casellas JM, Madhi SA, Urquhart TJ, Delport SD, Ferrero F et al. Impact of Haemophilus influenzae type $\mathrm{b}$ conjugate vaccine in South Africa and Argentina. Pediatr Infect Dis J 2004;23:842-7.

40. Morris SK, Moss WJ, Halsey N. Haemophilus influenzae type b conjugate vaccine use and effectiveness. Lancet Infect Dis 2008;8:435-43.

41. Scott JAG, English M. What are the implications for childhood pneumonia of successfully introducing $\mathrm{Hib}$ and pneumococcal vaccines in developing countries? PLoS Med 2008;5:e86.

42. Morgan G. What, if any, is the effect of malnutrition on immunological competence? Lancet 1997; 349:1693-5.

43. Balasubramanian V, Wiegeshaus EH, Taylor BT, Smith DW. Pathogenesis of tuberculosis: pathway to apical localization. Tuber Lung Dis 1994;75:168-78.

44. Schuster CA, Duffau TG, Eric NR, Mario PC. Lung aspirate punctureas a diagnostic aid in pulmonary tuberculosis in childhood. Pediatrics 1968;42:647-50.

45. Ahmed T, Sobhan F, Ahmed AMS, Banu S, Mahmood AM, Hyder KA, et al. Childhood tuberculosis: a review of epidemiology, diagnosis and management. Infect Dis J Pakistan 2008;17:52-60.

46. World Health Organization. Guidance for national tuberculosis programmes on the management of tuberculosis in children. Geneva: World Health Organization, 2006. $41 \mathrm{p}$.

47. Hatherill M, Hanslo M, Hawkridge T, Little F, Workman L, Mahomed $\mathrm{H}$ et al. Structured approaches for the screening and diagnosis of childhood tuberculosis in a high prevalence region of South Africa. Bull World Health Organ 2010;88:312-20.

48. Dye C. Global epidemiology of tuberculosis. Lancet 2006;367:938-40.

49. Hammitt LL, Murdoch DR, Scott JAG, Driscoll A, Karron RA, Levine OS et al. Specimen collection for the diagnosis of pediatric pneumonia. Clin Infect Dis 2012;54(Suppl 2):S132-9. 